mention along with ducted fans and other unconventional, vertical take-off aircraft.

European conditions may be sufficiently different from those in the United States and Australia for changes in aircraft, application techniques and organization of the work to be necessary, just as fertilizer and plant protection programmes differ. This report mainly poses the questions to which answers are needed to help the industry to prosper, and it is valuable for this alone. It is well produced, but an index would be helpful.

$$
\text { R. J. Courshet }
$$

\section{THEORY OF BANACH ALGEBRAS}

\section{General Theory of Banach Algebras}

By Prof. Charles E. Rickart. (The University Series in Higher Mathematics.) Pp. xi +394. (Princeton, N.J.: D. Van Nostrand Company, Inc.; London : D. Van Nostrand Company, Ltd., 1960.) $79 s$.

THIS book gives a comprehensive and detailed account of the theory of Banach algebras, and should be of great value to research workers in this field. The subject is a very modern one, the founda. tions for a general theory being laid by Gelfand in a paper published in 1941, but announced in 1939 (Normierte Ringe, Recueil Math. (Mat. Sbornik), N.S., 9,3 ; 1941).

The contents of the book are as follows. Chapter 1, "Fundamentals"; this includes definitions, the regular representations, complexification of a real normed algebra, the groups of regular and quasiregular elements, topological divisors of zero, the spectrum, and normed division algebras. Chapter 2, "The Radical, Semi-simplicity and the Structure Spaces" ; this includes ideals and difference algebras, representations, the radical, primitive Banach algebras, uniqueness of the norm topology and the fundarnental isomorphism theorem, structure of semisimple Banach algebras, the structure spaces, completely regular algebras and annihilator algebras. Chapter 3, "Commutative Banach Algebras"; this includes the carrier space and the Gelfand representa. tion theorem, algebras of functions, the Silov boundary, representations of the carrier space, homomorphisms of certain function algebras into a Banach algebra, direct-sum decompositions and related results and completely regular commutative Banach algebras. Chapter 4, "Algebras with an Involution" ; this includes miscellaneous properties of *-algebras, commutative *-algebras, self-dual vector spaces and *-representations, representations on Hilbert space, positive functionals and irreducible *-representations, symmetric *-algebras, general properties of B*. algebras, structure of ideals and representations of $\mathrm{B}^{*}$-algebras, and Banach*-algebras with minimal ideals.

There is, finally, a lengthy appendix on examples and applications, under three headings : $(a)$ "Algebras of Operators"; (b) "Algebras of Functions"; (c) "Group Algebras". Included in $(a)$ are the algebras g( the Schmidt-class $\%$, the trace-class $\%, W^{*}$ and $A W^{*}$ algebras, and spectral operators ; under (b) are included the algebra $C(\Omega)$ for certain special $\Omega$, the $l p$-algebras, functions with absolutely convergent Fourier series, functions of class $C^{(n)}$, continuous functions of bounded variation, holomorphic functions of one variable, holomorphic functions of several variables, a non-self-adjoint algebra with Silov boundary equal to the carrier space, non-existence of the Silov boundary, algebras of set functions on the line, some radical algebras, and algebras of power series; while (c) includes the algebra $L^{\mathfrak{l}}(\boldsymbol{g})$, locally compact abelian groups, group algebras of a compact group, almost periodic functions on groups, and group algebras of operators.

The book is evidently written for mature mathematicians who already have at least a slight acquaintance with the subject. Those not so equipped would, apart from the difficulty of the text, find the lack of simple illustrative examples in the four chapters a serious stumbling-block. The appendix would not supply this need, as the applications are given in a very condensed manner, requiring a high degree of maturity from anyone hoping to profit from it. However, for professional mathematicians having already some knowledge of the subject, the book will undoubtedly be of great value. R. G. Cooke

\section{DR. ERIC C. S. MEGAW}

\section{A Backroom Boy}

By Arthur Stanley. Pp. vi +106 . (Belfast: W. Erskine Mayne, Ltd., 1960.) 10s. 6d. net.

7 HIS little book, written by a father as a memorial to his brilliant son, describes the life of Dr. Eric C. S. Megaw, who was director of physical research at the Admiralty when he died at the early age of forty-eight years.

As a schoolboy Megaw acquired a sound knowledge of the French language, including facility in speaking it. Also, an interest in mechanical things led him at the age of fourteen to a keen interest in amateur radio experiments. This was encouraged by a short period in the Signals Section of the Officers' Training Corps, and in 1924 he was reputed to be the first amateur to send radio signals from Ireland. In the following year he established the first radio contact between Ireland and Australia.

The book outlines in the author's 'domestic' style Megaw's scientific career which was described in the obituary notices published in Nature (177, 409; 1956). The factual account is supplemented by extracts from his personal letters, which illustrate his acute power of observation and close attention to the work in hand. The final chapters of the book comprise extracts from letters received by Mrs. Megaw from officials and colleagues who were closely associated with, and very appreciative of, the scientific work of her late husband.

This volume is prefaced by a letter from Sir Eric Ashby, formerly vice-chancellor of the Queen's University of Belfast, where Megaw obtained both his bachelor's and doctoral degree in science. Sir Eric welcomes this memorial as a reassurance to both teachers and students, in describing "how the most, satisfying career can be built on quiet, steady work, without publicity, without ostentation, and without neglecting family life, or music, or literature".

A brief appendix lists Megaw's published papers, including that with two of his colleagues, which was published posthumously and was awarded the highest premium of the Institution of Electrical Engineers.

R. L. SMrth-Rose 